\title{
Effect of Phase Modulation of a Laser Pulse on the Generation of a Coherent Totally Symmetric Phonon in a Tellurium Single Crystal
}

\author{
O. V. Misochko ${ }^{a}$, S. V. Andreev ${ }^{b}$, V. O. Kompanets Ku. Y. Matveets $^{b}$, \\ A. G. Stepanov $b$, S. V. Chekalin ${ }^{b}$, and T. Dekorsy ${ }^{c}$ \\ ${ }^{a}$ Institute of Solid State Physics, Russian Academy of Sciences, Chernogolovka, Moscow oblast, 142432 Russia \\ e-mail:misochko@issp.ac.ru \\ ${ }^{b}$ Institute of Spectroscopy, Russian Academy of Sciences, Troitsk, Moscow oblast, 142190 Russia \\ ${ }^{c}$ Konstanz University, Physics Department, Konstanz, 78457 Germany
}

\begin{abstract}
The effect of phase modulation (resulting in a chirp of an ultrashort laser pulse) on the generation of a coherent $A_{1}$ phonon in Te was studied. The amplitude of coherent oscillations was found to depend on the sign and value of the pulse chirp: the oscillation amplitude decreases as the chirp increases. For a positive chirp, this effect is twofold stronger than for a negative one. The frequency-resolved response of a bandwidth-limited pulse was studied, which revealed the difference of oscillations and the relaxation response for the Stokes and anti-Stokes frequencies. The detected phenomena can be used for coherent control of lattice dynamics.
\end{abstract}

\section{INTRODUCTION}

At the end of the last century, the response of condensed media to exposure to an ultrashort laser pulse with energy concentrated in a small spatio-temporal interval began to be extensively studied. The access to the ultrafast dynamics of the lattice has been provided by observation of coherent phonons (CPs), which are manifested as oscillations in the light reflection after the crystal is exposed to an ultrashort laser pulse [1]. The use of high-energy laser pulses made it possible to achieve lattice displacement amplitudes at which nonlinear vibrations appear. These vibrations generate qualitatively new effects, many of which have not yet been completely comprehended [2-8]. In spite of the fact that many studies [1-8] have been directed to clarify the CP nature and the mechanisms of the formation of lattice subsystem coherence in crystals with large linear absorption, the effect of phase modulation of a laser pulse on the parameters of coherent oscillation has not been analyzed in those studies. The phase modulation leading to a chirp of the carrier frequency in the laser pulse (i.e., the time dependence of the "instantaneous" frequency of the electromagnetic field) has huge potential in studies of ultrafast lattice dynamics, since the use of coherent control methods allows separation of the effects of the laser pulse spectrum and rise rate on CP generation. This circumstance is due to the fact that the frequency chirp caused by phase modulation leads to changes in spatio-temporal characteristics of the laser pulse. One of these characteristics is the shape asymmetry (the leading edge becomes steeper, whereas the trailing edge elongates, or vice versa), because the long-wavelength components lead or lag the shortwavelength components, depending on the chirp sign. The existing gap in the study of the fast dynamics of opaque media with a long-range order (crystals) is striking, since the chirp effect has been successfully studied for a long time in molecular and physicochemical systems and the results are widely used to control the quantum states of molecules and chemical reactions [9]. Those studies have shown that the chirp has a significant effect on the amplitude of excited vibrational modes. By analogy, one might expect the possible effect of the laser pulse chirp on the parameters of coherent oscillations in crystals, especially at large (near-ultimate) amplitudes, at which the effects associated with oscillation anharmonicity begin to appear. This problem may be important in studying fast lattice dynamics, since ultrashort pulses have a broad spectrum and, hence, easily gain a positive chirp on their way to a sample when passing through a system of lenses, beam splitters, attenuators, or cryostat windows. In addition to its practical importance, the study of the generation of CPs by pulses with a controlled chirp can yield additional information on the lattice coherence mechanism itself and thus provide a solution to the widely discussed problem of $\mathrm{CP}$ generation in opaque media [1-8]. The goal of this study is to fill this gap, 
i.e., to begin a detailed study of the dependence of coherent oscillations on the laser pulse chirp value and sign. To our knowledge, we are the first to experimentally demonstrate the effect of the femtosecond laser pulse chirp on the amplitude of coherent oscillations in a tellurium crystal at high excitation levels close to the ultimate one.

\section{EXPERIMENTAL}

Tellurium was chosen as a sample for studying the effect of the chirp on coherent lattice dynamics. This narrow-bandgap semiconductor can be a model system, since its electron and phonon subsystems have been studied theoretically and experimentally in sufficient detail, by using, in particular, the method of pumping and probing with ultrashort pulses [2, 3, 8]. Tellurium crystallizes in the $D_{3}^{4}$ structure in which three unit-cell atoms form a helical chain turn oriented along the trigonal axis. The normal vector of the totally symmetric $A_{1}$ phonon corresponds to atomic motion changing the helix radius and retaining the lattice symmetry.

The effect of the chirp on the amplitude of coherent oscillations is studied by the standard pump-probe method using laser pulses with a wavelength of $800 \mathrm{~nm}$, generated as a result of amplification of pulses from a master oscillator (Spectra Physics, "Tsunami") in a Ti : sapphire-crystal regenerative amplifier (Spectra Physics, "Spitfire"). The repetition rate of laser pulses in the output of the amplifier was $1 \mathrm{kHz}$, and the pulse energy was $\approx 0.7 \mathrm{~mJ}$. After attenuation to a required level, the excitation and probe beams with an intensity ratio of about 20:1 were focused using thin quartz lenses and were brought together at an angle of $\approx 4^{\circ}$ in the region under study on the crystal plane containing the trigonal and bisector axes. The spot diameters of excitation and probe beams at the point of their interaction with the sample were about 100 and $40 \mu \mathrm{m}$, respectively. The beam polarization planes were mutually perpendicular, which allowed a significant reduction of the effect of the scattered light from the excitation beam by using a crossed polarizer positioned before a photodetector detecting probe radiation. In the experiments, the difference reflectance of the probe radiation (with and without excitation beam pulse) was measured. To this end, the excitation beam was modulated using a mechanical chopper with a duty ratio equal to unity and a frequency of $500 \mathrm{~Hz}$. CP dynamics was studied by introducing a tunable optical delay line (whose delay time could be changed in steps of $\approx 1 \mathrm{fs}$ ) into the excitation beam. The value and sign of the laser pulse chirp were varied by detuning the pulse compressor in the femtosecond amplifier with respect to the position corresponding to a minimum duration of bandwidth-limited pulses coming out from the amplifier. A positively chirped pulse has low-frequency components at the leading edge and high-frequency components at the trailing edge. For a negatively chirped pulse, the situation is reversed. The chirp was estimated by the change in the pulse duration. The duration of pulses shorter than 100 fs was measured using an autocorrelator. Larger pulse durations were estimated from the pulse compressor detuning time (the operating time of the step motor in the femtosecond amplifier) with respect to the position corresponding to a minimum duration of pulses coming out from the amplifier. The value of the spectral chirp $\alpha$ was estimated from the formula $\alpha=$ $\pm 0.36 \tau_{0} \sqrt{\tau_{\mathrm{c}}^{2}-\tau_{0}^{2}}$ (valid for Gaussian pulses), where $\tau_{0}$ is the duration of a bandwidth-limited pulse and $\tau_{\mathrm{c}}$ is the duration of the chirped pulse. The influence of the chirp on the amplitude of coherent oscillations was observed for the excitation beam intensity close to the ultimate one, which was experimentally determined by the absence of degradation of the reflectance of the sample over the time of one cycle of measurement of signal kinetics (approximately $20 \mathrm{~min}$ ). All measurements of the ultrafast lattice dynamics of the tellurium single crystal were carried out at room temperature. The zero delay was determined by the maximum derivative of the reflection signal.

\section{RESULTS AND DISCUSSION}

Figure 1 shows the typical optical response observed during excitation followed by probing the tellurium single crystal by laser pulses differing in the value and sign of the frequency chirp. In all cases, the excited crystal state relaxes to an equilibrium state in times of the order of ten picoseconds; fast oscillations caused by the totally symmetric $A_{1} \mathrm{CP}[2,6,8]$ are superimposed on this relaxation. Differences are observed in the value of the photoinduced signal, the ratio of the oscillatory and relaxation (not oscillating) signal components, and the coherent oscillation amplitude. The last effect becomes more obvious after numerically subtracting the relaxation component, which is usually attributed to electronic excitations. The result of this processing is shown in Fig. 2. The oscillation component is fitted by a damping harmonic function $A \exp (-t / \tau) \sin (2 \pi \nu+\phi)$, which allows one to determine the main oscillation parameters (an alternative method is an analysis of the spectrum of the Fourier-transformed signal, which was also used and yielded identical results). It is clear that the oscillation amplitude depends on the chirp value and sign. An increase in the amplitude in the case of excitation by bandwidth-limited pulses is natural, since a shorter pulse provides a higher peak field strength. However, the difference for pulses differing in chirp sign and having approximately equal durations cannot to be explained in these terms. As the chirp magnitude is increased, the rate of decrease in the oscillation amplitude for positive chirps is twice that for negative chirps. This asymmetry (Fig. 3) suggests that positive and neg- 

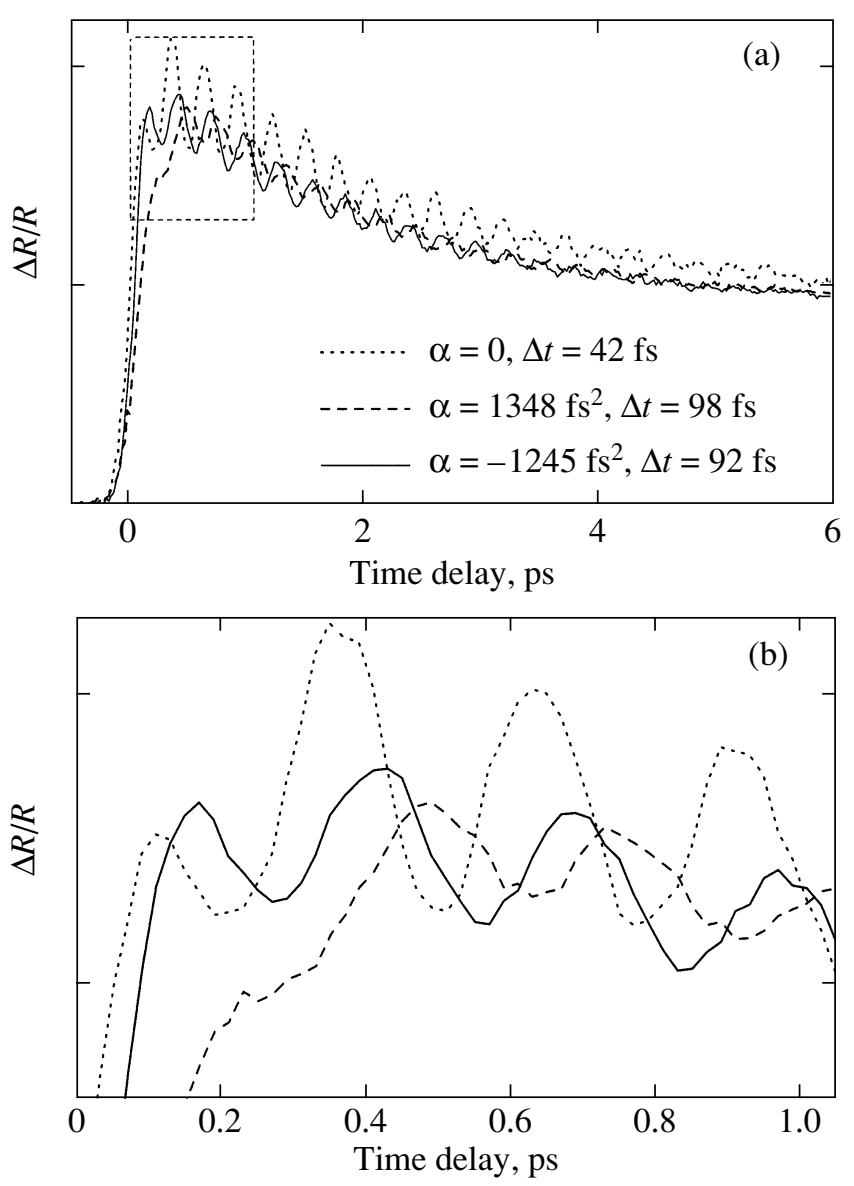

Fig. 1. (a) Dependence of the relative difference reflectance $\Delta R / R=[R(t)-R(t<0)] / R(t<0)$ of a probe pulse for a Te single crystal on the time delay between the pump and probe pulses for various values of the laser pulse chirp $\alpha$ (the pulse duration $\Delta t$ is indicted for each chirp). (b) Oscillations at small time delays on a larger scale.

ative chirps are nonequivalent; therefore, the observed effect is not associated with an increase in the pulse duration. It is clear that, under the conditions of this experiment, negatively chirped pulses are more efficient in producing lattice coherence (i.e., provide a larger oscillation amplitude) in comparison with positively chirped pulses of the same duration.

As the laser pulse chirp increases in magnitude, the oscillation frequency remains unchanged to within the experimental error and coincides with that caused by the bandwidth-limited pulse. For positive chirps, an insignificant $(\sim 15 \%)$ increase in the oscillation lifetime is observed with respect to almost coinciding lifetimes of phonons excited by negatively chirped bandwidthlimited pulses. It also follows from Fig. 1 that, for chirped pulses, oscillations are observed at slightly longer delay times and this effect is most pronounced for positive chirps. As the chirp increases, this time shift increases, which can be seen in Fig. 4. The signal rise becomes much slower for the positive chirp, and

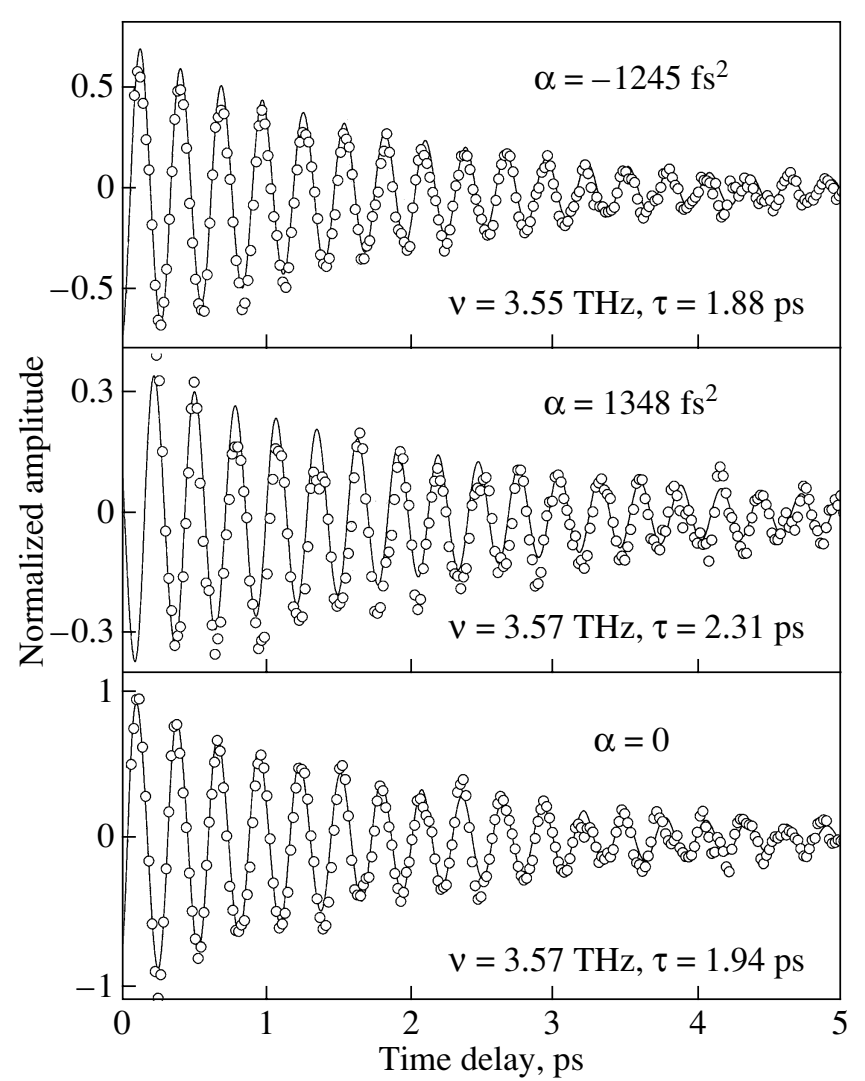

Fig. 2. Probe signal oscillations for various chirps (dots) and fitting of the data by harmonic damped oscillations (solid curves). The oscillation frequency $v$ and damping time $\tau$ obtained by fitting are indicated.

oscillations (decreased in amplitude) appear at longer time delays. This effect cannot be explained in terms of a possible shift of the time delay zero, which is determined using the extremum of the signal rise derivative for the bandwidth-limited pulse. Indeed, the zero delay for the chirped pulse, defined as the time coincidence of pump and probe pulses, can vary due to a change in the pulse shape; however, this time shift cannot be longer than the pulse duration and has opposite signs for positive and negative chirps. According to the experimental data, the time shift has the same sign irrespective of the chirp sign and, for the positive chirp, is severalfold larger than the laser pulse duration.

The laser pulse chirp changes both the time sequence of various spectral components of the electromagnetic field (which is equivalent to an increase or a decrease in the carrier frequency) and the pulse shape. However, the mere fact that the coherent oscillation parameters depend on the chirp does not allow a unambiguous choice between two phenomenological models of CP generation in opaque media $[1,10,11]$. Indeed, an increase in the oscillation amplitude can be caused by both the steeper leading edge and the fact that the high-frequency components of the carrying frequency interact with the crystal earlier than do the low-fre- 


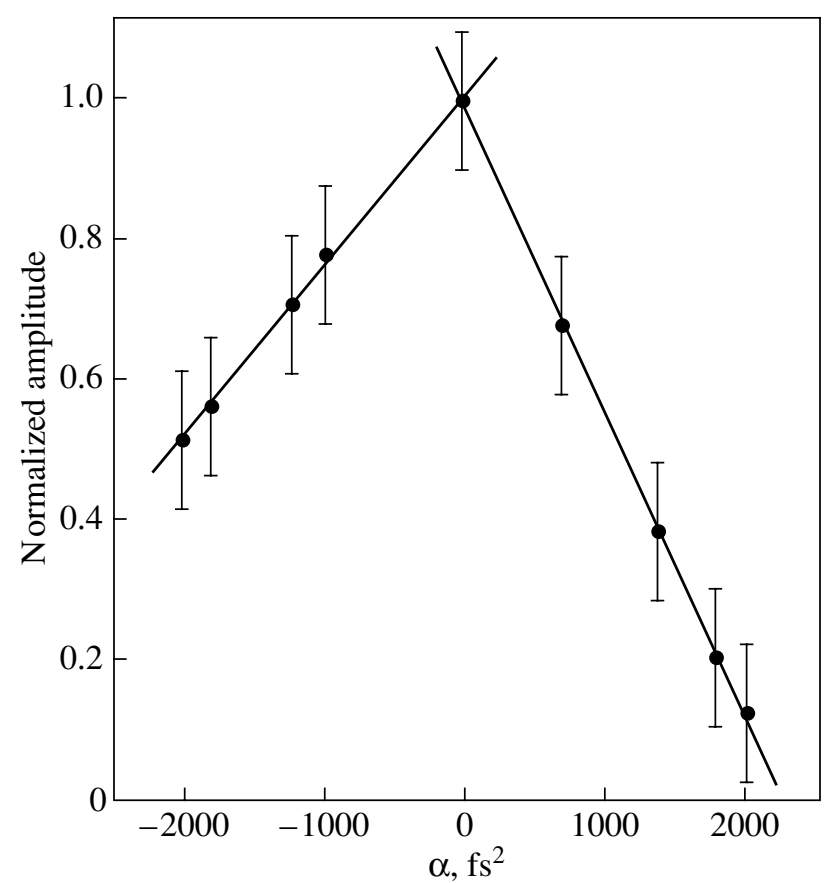

Fig. 3. Dependence of the normalized coherent oscillation amplitude on the laser pulse chirp. Straight lines are fits (separately for positive and negative chirps).

quency components. The main difference between the two basic phenomenological models of CP generation is the role played by the electromagnetic field. In the shift mechanism (SM), the field provides only energy required to excite the system, which is introduced through the electron subsystem of the crystal and is transferred to the lattice via the electron-phonon interaction. In this model, the photon-electron and electron-phonon interactions are separated and the former interaction is not explicitly defined. The single parameter of the photon-electron interaction is the rate at which it is turned on, which should be so high that the characteristic time of the photon-electron interaction would be shorter than the phonon oscillation period. As a result, lattice coherence always occurs in an excited electronic state and the lattice exposed to an ultrashort pulse evolves following a cosine law; i.e., oscillations begin when the amplitude has an extremum value (maximum or minimum). In the mechanism of nonsteady-state stimulated Raman scattering (NSRS), the initial phonon phase and symmetry are uniquely defined by the relation between the components of the electromagnetic field spectrum and the properties (resonances) of the medium [1]. In this model, the photon-electron and electronphonon interactions are inseparable and lattice coherence can occur in both an excited (resonant case) and the ground (nonresonant case) electronic state. Thus, a criterion for NSRS is the width of the excitation pulse spectrum for which two-photon

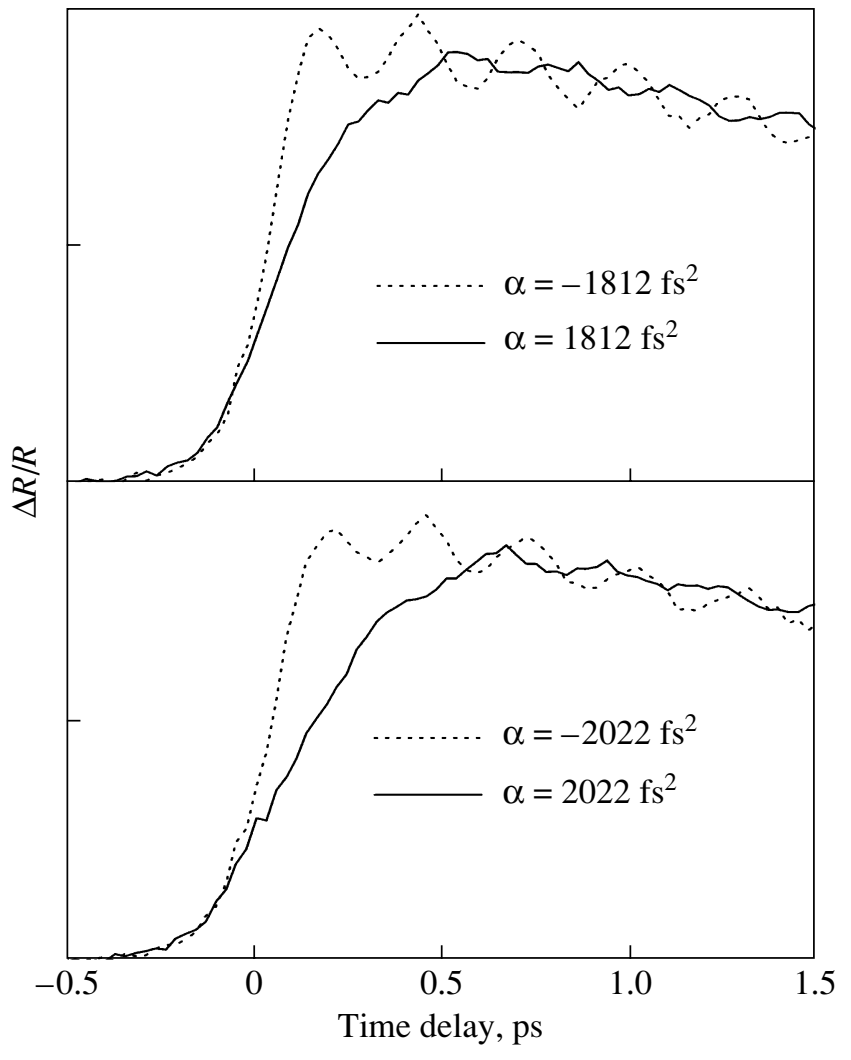

Fig. 4. Dependence of the relative difference reflectance $\Delta R / R$ of a probe pulse for a Te single crystal on the time delay between pump and probe pulses at large values of the laser pulse chirp.

pumping becomes possible (i.e., the difference between various components of the pulse spectrum should be equal to or larger than the phonon frequency).

In order to study lattice dynamics for short time delays, which in principle could allow one to separate the effects of the leading edge steepness and the time sequence of different components of the pulse spectrum, we cannot employ the above procedure of chirp introduction, since the chirp is introduced before the pulse is split into pump and probe, which inevitably lengthens both the pump and probe pulses. Thus, we cannot study the kinetics of reflection signal rise in order to clarify the role played by the steepness of the leading edge of the excitation pulse. If only the pump pulse is chirped, then probing with much shorter bandwidth-limited pulses will become possible. However, we can obtain additional information on the $\mathrm{CP}$ generation mechanism by analyzing the spectrally-resolved response of a probe pulse. To this end, before recording the probe pulse, its spectral decomposition was performed using a spectrometer and then the frequencyresolved signal was analyzed using a multichannel detector. The results obtained for a bandwidth-limited pulse are shown in Fig. 5. It is clear that the Stokes and 


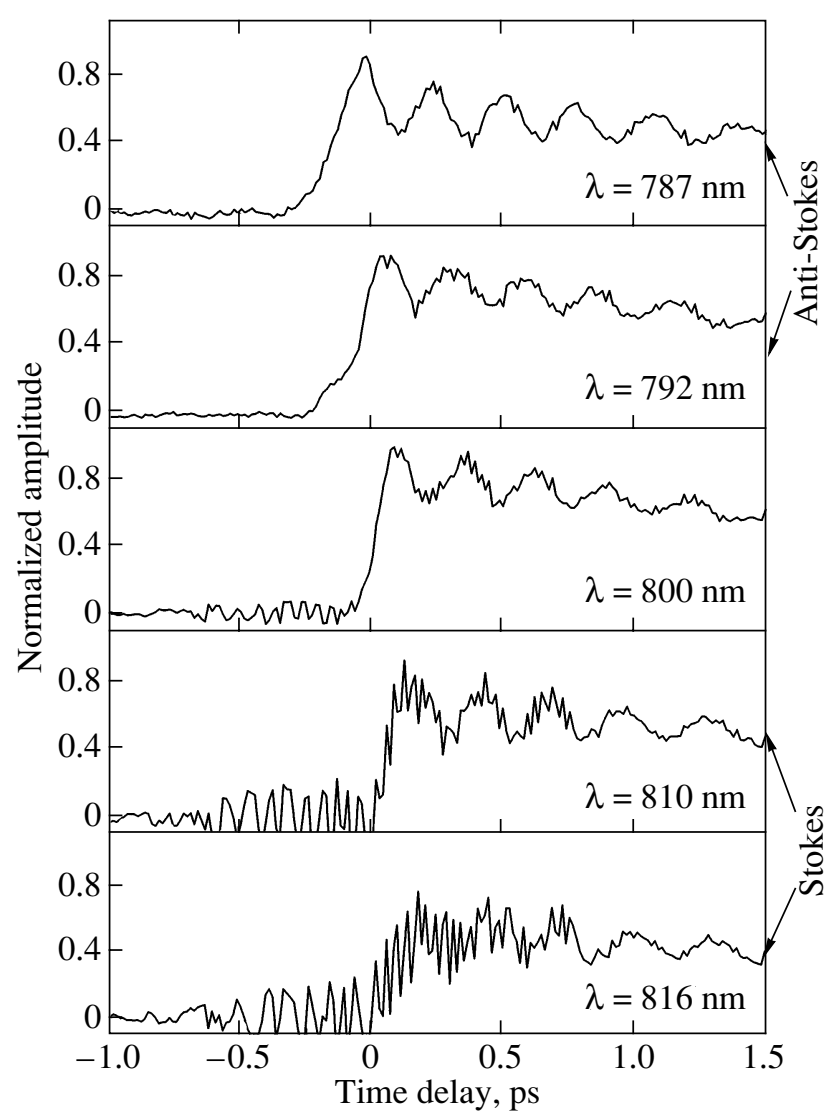

Fig. 5. Normalized (to unity at $\lambda=800 \mathrm{~nm}$ ) frequencyresolved reflectance of a Te single crystal for various wavelengths (indicated in the panels) of a probe bandwidth-limited pulse.

anti-Stokes components of the probe pulse are phaseshifted, which confirms the results of single-channel detection of CPs in semimetals [12]. It should also be noted that, as the detuning from the central carrier frequency of the pulse to either short or long wavelengths increases, the oscillation visibility defined as $\left(A_{\max }-\right.$ $\left.A_{\min }\right) /\left(A_{\max }+A_{\min }\right)$ increases almost twofold. This suggests that the contribution of the relaxation component decreases as the detuning increases. Unexpected is the fact that the Stokes signal contains strong noise, which is also detected at negative time delays $\Delta t \approx-500 \mathrm{fs}$ (we recall that the laser pulse duration is $42 \mathrm{fs}$ ). This noise is also observed for positive time delays $\Delta t \approx 800 \mathrm{fs}$, after which the noise level for the Stokes signal becomes equal to that observed for the anti-Stokes signal. The increase in noise at the Stokes frequency is naturally explained in terms of NSRS. In the anti-Stokes process, the final state is vacuum, whereas in the Stokes process the final state is the first excited state of the lattice, in which the noise level is proportional to the Bose factor and, hence, can be higher. We note that the appearance of noise at negative time delays (i.e., in the case where the probe pulse comes earlier than does the excitation pulse) is in many respects similar to the appearance of a signal at negative time delays in experiments on four-wave mixing in semiconductors, where this fact was interpreted as the effect of many-particle Coulomb correlations, leading to violation of the causality principle [13].

The differences in the responses at the Stokes and anti-Stokes frequencies of the probe pulse count in favor of the NSRS mechanism, since the shift mechanism produces a response uniform in frequency (in this mechanism, the lattice system gains energy irrespective of the frequency of the driving force). Only in NSRS can the lattice both gain (Stokes process) and lose (antiStokes process) energy, which naturally leads to a frequency dependence of the probe pulse signal.

\section{CONCLUSIONS}

Using the method of pumping and probing with femtosecond laser pulses with varying frequency chirp, we have detected the dependence of the coherent oscillation amplitude on the chirp sign. This dependence suggests that negative chirps cause more efficient generation of coherent phonons (CPs). From a combined analysis of the data obtained using chirped pulses and of the results of studying the frequency-resolved response for bandwidth-limited zero-chirp pulses, the conclusion has been drawn that the $\mathrm{CP}$ generation mechanism involves various components of the electromagnetic field spectrum and, hence, is associated with two-photon pump.

\section{ACKNOWLEDGMENTS}

The authors are grateful to Yu.E. Lozovik for helpful discussions.

This study was supported by the Russian Foundation for Basic Research (project nos. 06-02-16186-a, 07-02-00148, and 05-02-17338-a) and the German Research Foundation (Deutsche Forschungsgemeinschaft, Grant DE 567/9).

\section{REFERENCES}

1. R. Merlin, Solid State Commun. 102, 207 (1997).

2. S. Hunsche, K. Wienecke, T. Dekorsy, and H. Kurz, Phys. Rev. Lett. 75, 1815 (1995).

3. P. Tangney and S. Fahy, Phys. Rev. B: Condens. Matter 65, 054302 (2002).

4. M. Hase, M. Kitajima, S. Nakashima, and K. Mizoguchi, Phys. Rev. Lett. 88, 067401 (2002).

5. O. V. Misochko, M. Hase, and M. Kitajima, Pis'ma Zh. Éksp. Teor. Fiz. 78 (2), 85 (2003) [JETP Lett. 78 (2), 75 (2003)]. 
6. C. A. D. Roeser, M. Kandyla, A. Mendioroz, and E. Mazur, Phys. Rev. B: Condens. Matter 70, 212302 (2004).

7. O. V. Misochko, M. Hase, K. Ishioka, and M. Kitajima, Pis'ma Zh. Éksp. Teor. Fiz. 82 (8), 478 (2005) [JETP Lett. 82 (8), 426 (2005)].

8. É. D. Murray, D. M. Fritz, J. K. Wahlstrand, S. Fahy, and D. A. Reis, Phys. Rev. B: Condens. Matter 72, R060 301 (2005).

9. M. Dantus and V. V. Lozovoy, Chem. Rev. (Washington) 104, 1813 (2004).
10. G. A. Garrett, T. F. Albrecht, J. F. Whitaker, and R. Merlin, Phys. Rev. Lett. 77, 3611 (1996).

11. H. J. Zeiger, J. Vidal, T. K. Cheng, E. P. Ippen, G. Dresselhaus, and M. S. Dresselhaus, Phys. Rev. B: Condens. Matter 45, 768 (1992).

12. O. V. Misochko, M. Hase, and M. Kitajima, Fiz. Tverd. Tela (St. Petersburg) 46 (9), 1686 (2004) [Phys. Solid State 46 (9), 1741 (2004)].

13. D. S. Chemla and J. Shah, Nature (London) 411, 549 (2001)

Translated by A. Kazantsev 